\title{
Weltstädte
}

\section{Einige neue Hypothesen zur Dynamik und Struktur von Weltstädten}

\section{Zur Thematik}

Aufschwung und Niedergang von Weltstädten sind allgemein stark beachtete und oft diskutierte Phänomene. Die Frage, warum ehemals glanzvolle Metropolen des 19. Jahrhunderts wie Konstantinopel, Neapel und Kairo heute weitgehend nur noch nationale Ausstrahlungskraft besitzen, während London und Paris auch im 21. Jahrhundert von überragender weltweiter Bedeutung sein werden, hat immer wieder Wissenschaftler und Intellektuelle beschäftigt. Schlüssige Antworten wurden bislang allerdings kaum gefunden.

Um es gleich vorwegzunehmen: Auch der vorliegende Artikel läßt zahlreiche Fragen offen und wirft neue auf. Der neu entwickelte theoretische Ansatz liefert jedoch Anhaltspunkte, wie die Fragestellung angesichts der sich immer rascher wandelnden regionalwirtschaftlichen und politischen Bedingungen angepackt werden könnte und wo Bedarf nach weiterführender Analyse besteht.

Dieser Artikel basiert auf einem Kleinprojekt im Rahmen des Nationalen Forschungsprogrammes "Stadt und Verkehr».' In der Form eines wissenschaftlichen Essays wurde darin sowohl theoretisch als auch empirisch der Frage nachgegangen, inwiefern sich Struktur und Dynamik von Weltstädten vor dem Hintergrund des dynamischen weltwirtschaftlichen Strukturwandels verändern. Darüberhinaus wurde die Rolle der wichtigsten Schweizer Städte im Weltstädte-System analysiert.

Das Schwergewicht des vorliegenden Artikels liegt auf der theoretischen Diskussion. In einem ersten Schritt werden einige ausgewählte Konzepte zum Thema Stadtentwicklung vorgestellt, welche in der Folge zu einem neuen theoretischen Ansatz verdichtet werden. Einige ausgewählte Ergebnisse der empirischen Untersuchungen und die daraus zu ziehenden Schlußfolgerungen runden diesen Artikel ab.

\section{Traditionelle stadtentwicklungstheoretische Ansätze}

Seit den Anfängen der Industrialisierung und Urbanisierung im 18. Jahrhundert beschäftigt das Thema Stadtentwicklung Wissenschaftler verschiedenster Fakultäten. Während in der Anfangsphase eher das rein wissenschaftliche Interesse im Vordergrund stand, kommt der Problematik eine zunehmende Bedeutung für Umwelt, Politik und Wirtschaft zu. So hat die Verstädterungsquote zwischen 1940 und 1980 in Industrieländern um 230\%, in Entwicklungsländern gar um $540 \%$ zugenommen. Dies führte dazu, daß 1980 schon jeder siebte Erdenbewohner in einer Millionen-Stadt und knapp 57\% der Weltbevölkerung in Städten mit mehr als 20000 Einwohnern lebten (STIGLBAUER, 1986: 15).

Die Stadtentwicklungsproblematik interessiert jedoch nicht nur aufgrund der demographischen Aspekte. Auch wirtschaftlich nimmt die Bedeutung der Städte zu; die den Gang der Weltwirtschaft prägenden Entscheidungen werden immer mehr in einigen wenigen globalen Zentren gefällt. Innerhalb des globalen Stadt-Systems zeichnete sich dabei bisher zunehmend eine Hierarchisierung ab.

Wie die folgenden Ausführungen zeigen werden, decken die bestehenden stadtentwicklungstheoretischen Ansätze zumeist nur einzelne Aspekte der Problematik ab. Im Kapitel 3 wird deshalb ein neuer Ansatz skizziert, welcher einerseits einen Erkenntnisfortschritt anbietet, andererseits als Plattform für weiterführende theoretische und empirische Studien dienen soll.

\subsection{Zentralörtliche Aspekte der Stadtentwicklung}

W. Christaller, der Begründer der Theorie der zentralen Orte, versuchte schon 1933, die hierarchische Struktur der räumlichen Ordnung der Wirtschaft und die Hierarchie der Siedlungen aus dem Zusammenwirken ökonomischer Bestimmungsfaktoren zu erklären und abzuleiten. Aufgrund verschiedener Überlegungen, welche hier nicht im einzelnen dargelegt werden sollen, kam Chri-

Thomas Kärcher; Brugger, Hanser und Partner, Winterthurerstr. 52, 8006 Zürich

\footnotetext{
1 Brugger Ernst A./Kärcher Thomas, 1992: Weltstädte Schweizer Städte: Die Funktion schweizerischer Großstädte im weltwirtschaftlichen Kontext. In: Bericht 5 des NFP "Stadt und Verkehr" (eine Studie von BRUGGER, HANSER UND PARTNER), Zürich.
} 
staller unter anderem zu folgenden Schlüssen (nähere Erläuterungen in: SCHÄTZL, 1981: 69):

- Jedes Gut (Waren, Dienstleistungen) hat seine eigene Reichweite. Die Zentralität eines Gutes wird durch die Nachfrage nach diesem Gut bestimmt. Je mehr zentrale Güter an einem Standort angeboten werden, desto höher ist die Zentralität des Ortes.

- Das räumliche Verteilungsmuster der zentralen Orte und ihrer Marktgebiete hängt von der Reichweite der zentralen Güter ab.

- Zentrale Orte einer bestimmten Ordnung bieten nicht nur jene Güter an, die ihrem Zentralitätsrang entsprechen, sondern auch alle Güter der Orte niederer Ordnung. Alle zentralen Orte gleicher Ordnung sind funktional identisch; sie weisen keine wesentlichen Unterschiede hinsichtlich der Zahl der angebotenen Güter, der Struktur des Warenkorbes und der Struktur des Marktgebietes auf.

- Ein zentraler Ort höchster Ordnung und alle innerhalb seines Marktgebietes liegenden zentralen Orte und Marktgebiete niederer Ordnung stellen ein geschlossenes funktionales System dar.

In seiner Theorie der Marktnetze versucht A. Lösch (1940), die räumliche Verteilung der Produktionsstandorte sowie die räumliche Produktionsspezialisierung zu erklären. Aufgrund seiner Annahme, daß für jedes Gut ein produktionsspezifisches Marktgebiet besteht, zieht Lösch den Schluß, daß für jedes Gut ein Netz von Marktgebieten mit charakteristischen Maschengrößen existiere. Dieses System von Marktnetzen stellt für Lösch das "Idealbild einer Wirtschaftslandschaft» dar, und er nimmt an, daß sich solche Systeme netzförmig über die Erde verteilen (vgl. dazu SCHÄTZL, 1981: 73-79).

Die Vorstellung der netzförmig über die Erde verteilten Marktgebiete taucht in verschiedenen Abhandlungen zur Stadtentwicklungsproblematik wieder auf (vgl. dazU FRIEDMANN, 1986). Der Gedanke, wonach sich ein zentraler Ort durch die Wahrnehmung spezieller Funktionen von seinem Umfeld abhebt, wird in Kapitel 3 wieder aufgenommen.

\subsection{Neoklassische Konzeption der Stadtentwicklung}

Gemäß neoklassischem Verständnis nehmen Städte im allgemeinen Entwicklungsprozeß in erster Linie eine wachstumsfördernde Rolle wahr, indem sie Wachstumsimpulse für die umliegenden Gebiete vermitteln und somit zu einer gleichmäßig über den Raum verteilten Entwicklung führen. Dementsprechend konzentrierte sich die neoklassische Diskussion hauptsächlich auf die Frage, welches die für die Generierung von regionalen Wachstumsimpulsen optimale Stadtgröße ist. In verschiedenen Studien wurde die optimale Stadtgröße zur Wahrnehmung der urbanen Multiplikatorfunktion aufgrund wohlfahrtstheoretischer Faktoren, ökonomischer Effizienzkriterien und aufgrund des Verlaufs sozialer Grenzkosten zu ermitteln versucht.

Diese Fragestellung ist insbesondere vor dem Hintergrund zunehmender Umweltbelastungen hochaktuell.
Hohe Bevölkerungskonzentrationen entstehen dort, wo starke positive externe Effekte vorhanden sind oder zumindest perzeptiert werden. Bevölkerungsballungen ihrerseits vermögen externe Effekte zu verstärken. Positive externe Effekte sind Agglomerationsvorteile (insbesonders überdurchschnittlich attraktive Infrastrukturen), negative verursachen Agglomerationskosten, wie beispielsweise eine zunehmende Lärmbelästigung und andere Umweltbeeinträchtigungen, der hohe Zeitverlust beim Pendeln vom Wohnort zum Arbeitsort, soziale Unrast und viele andere mehr.

Die Tatsache, daß beispielsweise bei Umweltgütern der Steuerungsmechanismus über den Preis nicht oder nur unvollkommen funktioniert, ist auf die weitgehend kostenlose Nutzung dieser Güter zurückzuführen. «Da die Umweltnutzer in der Regel nichts für das Gut Umwelt zahlen müssen, stehen sie unter dem Eindruck, dieses sei ein freies, das heißt ein nicht knappes Gut. Für Ökonomen ist es überhaupt nicht überraschend, daß dieser Nulltarif zu einer Übernutzung der Umwelt führt und dadurch die Regenerationskraft der Natur überfordert wird» (FREY, 1990: 228). Diese Problematik akzentuiert sich in Stadtgebieten insofern, als die Wahrscheinlichkeit, daß externe Kosten auftreten und daß diese ein ins Gewicht fallendes Ausmaß annehmen, in der Regel umso größer ist, je dichter die Wirtschaftssubjekte zusammen leben (FREY, 1990: 220). Da zudem ein großer Anteil der gesamtwirtschaftlichen Kosten nicht von den Verursachern und auch nicht von den Entscheidungsträgern der Standortwahl von Unternehmen getragen wird, besteht die Wahrscheinlichkeit, daß die effektive StadtgröBe nicht dem volkswirtschaftlichen Optimum, sondern der Summe der individuellen, einzelwirtschaftlichen Optima entspricht. Städte tendieren deshalb dazu, ihre optimale Größe zu überschreiten.

Abbildung 1 stellt diesen Zusammenhang her und verknüpft die individuelle mit der kollektiven Nutzen- bzw. Lastenbeurteilung (vgl. WACHTER, 1990: $84 \mathrm{ff}$.).

In Abbildung 1 stellt $\mathrm{P}$ die Agglomerationsgröße (z. B. gemessen an der Bevölkerung) dar. Auf der Ordinate sind die Kosten und Nutzen aus der Zusammenballung abgebildet. PGK ist die private Grenzkostenkurve; sie stellt gleichzeitig die sozialen Durchschnittskosten aus der Zusammenballung, die jeder Stadtbewohner in Kauf zu nehmen hat, dar. Berücksichtigt man die externen Ballungskosten, so resultiert die soziale Grenzkostenkurve SGK. Spiegelbildlich verhält sich die Argumentation für die private und die soziale Grenznutzenkurve.

Das Modell erlaubt die Bestimmung von gruppenspezifisch optimalen Stadtgrößen. Bereits ansässige Bewohner bevorzugen die Stadtgröße E, weil hier die Differenz zwischen Durchschnittskosten und Durchschnittsnutzen maximal ist. Stadtgröße P' stellt das gesamt-gesellschaftliche Optimum dar, da hier die sozialen Grenzkosten gleich dem sozialen Grenznutzen sind. Da für $\mathrm{Zu}-$ wanderer allerdings wegen der Externalitätenproblematik nicht die ihnen zurechenbaren sozialen Grenzkosten und Grenznutzen relevant sind, sondern lediglich die 
sich ihnen offenbarenden Durchschnittswerte, besteht ein Anreiz, über das soziale Optimum hinaus bis zum Punkt P, der Marktlösung, in die Stadt zu ziehen, wo die vom Einzelnen zu tragenden privaten Grenzkosten gleich dem privaten Grenznutzen sind.
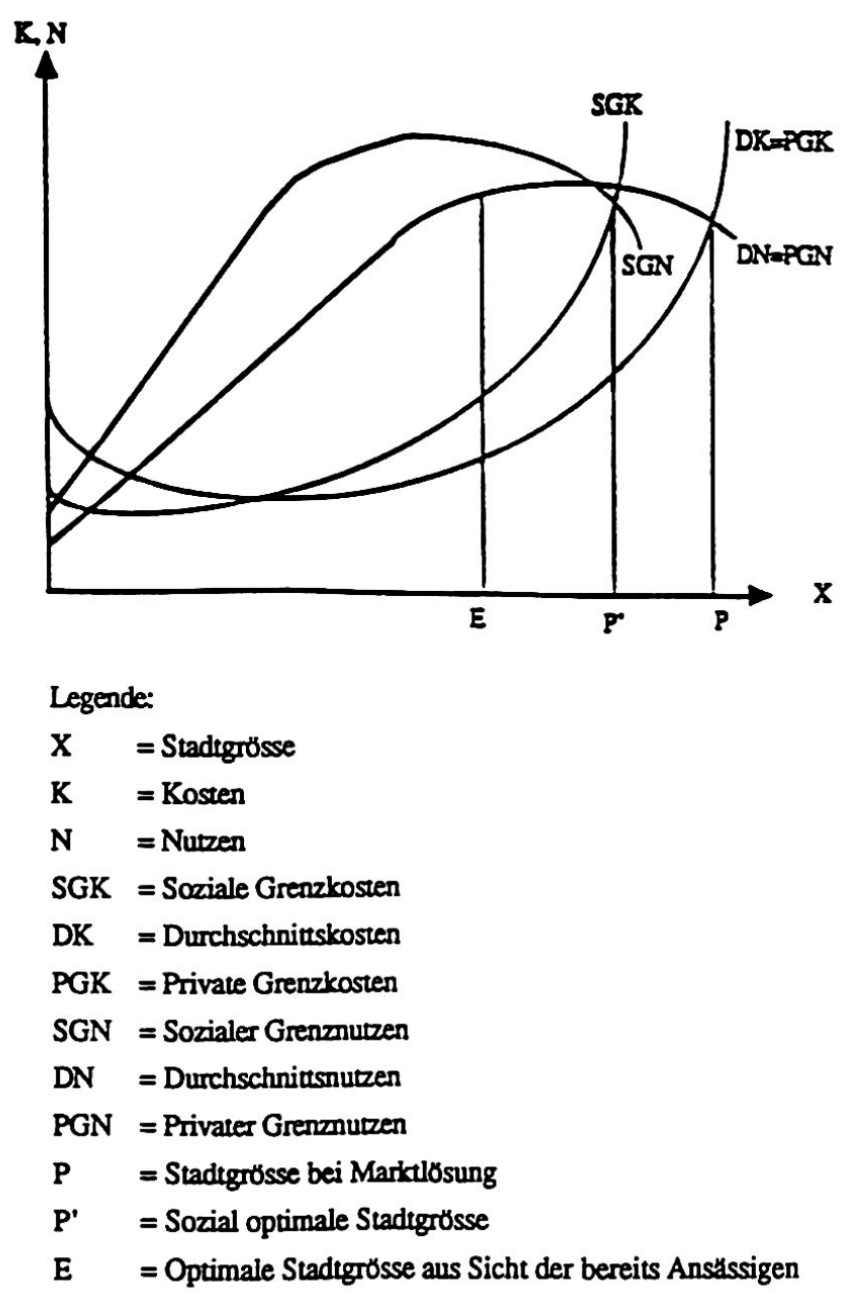

Quelle: Richardson 1978, 74

Abb. 1 Theorie der optimalen Stadtgröße.

\subsection{Polarisationstheoretisches Konzept der Stadtentwicklung}

Die polarisationstheoretischen Ansätze gehen, im Gegensatz zu den neoklassischen Theorien, grundsätzlich davon aus, daß sich die räumlichen Disparitäten im Laufe des Entwicklungsprozesses verstärken resp. daß Entwicklung nicht gleichgewichtig verläuft. Sektorale und regionale Ungleichgewichte sind somit eine logische Folge von wirtschaftlichem Wachstum und technischem Fortschritt.

Interessante Aspekte für das Verständnis der Stadtentwicklungsproblematik finden wir insbesondere im inno- vationstheoretisch fundierten Modell sektoral und räumlich polarisierter Entwicklung nach J. R. LASUÉN aus dem Jahre 1973. Darin wird behauptet, daß wirtschaftliche Entwicklung durch Anwendung von Innovationen entsteht und daß diese letzteren wiederum hauptsächlich im Umfeld von dominierenden Industrien auftreten und sich dort geographisch häufen. Für LASUÉN (1973: 164-176) sind räumliche Entwicklungs- und Urbanisierungsmuster die zeitlichen Spuren des Innovations-Adoptions-Prozesses. Innovationen treten dabei gehäuft (in Clusters) auf; sektorale und urbane Innovationsprozesse verlaufen deshalb nicht kontinuierlich. Sektorale Clusters sind für Lasuén Industriebetriebe und die Gesamtheit ihrer Lieferanten und Abnehmer. Ein sektoraler Cluster besteht aus Komplementaritäten, die sich im Laufe des Produktionsprozesses zwischen der Industrie und ihren Lieferanten resp. Abnehmern ergeben. Sektorale Clusters entstehen in der Regel in einem innovativen Umfeld, in sog. Innovations-Clusters. Innovationen wiederum, als internationale Neuheiten verstanden, basieren auf Erfindungen, welche wegen des großen Arbeitsmarktes, der guten Finanzierungsmöglichkeiten, der guten Ausbildungs- und Forschungsmöglichkeiten, aber auch wegen der hohen Bevölkerungsdichte hauptsächlich in Ballungsgebieten auftreten. Geographische Clusters sind einerseits räumliche Konzentrationen von Industrien und Firmen, andererseits allerdings auch «Bevölkerungs-Anhäufungen» und somit Voraussetzung für Innovations-Clusters. Vereinfachend können die eben beschriebenen Sachverhalte als System zirkulär verursachter kumulativer Prozesse dargestellt werden:

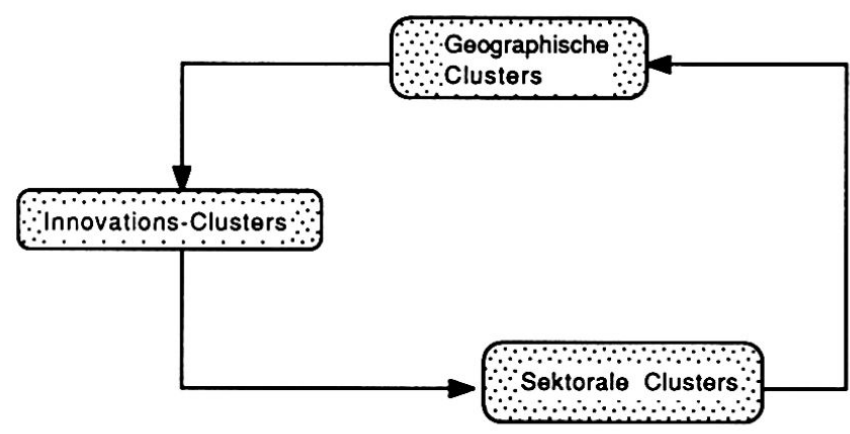

Abb. 2 Sektoral und räumlich polarisierte Entwicklung als Ergebnis eines zirkulär verursachten kumulativen Prozesses. (Quelle: Eigene Darstellung nach Lasuén.)

In diesem System spielen sich insofern kumulative Prozesse ab, als erstens die Komplementarität zwischen Innovationen zu stärker konzentrierten Innovations-Clusters führt und zweitens die «Umlaufgeschwindigkeit» im System ständig wächst. Zu Beginn des 20. Jahrhunderts betrug die Zeitdifferenz zwischen aufeinanderfolgenden industriellen Innovationen 37 Jahre, nach dem zweiten Weltkrieg noch 14 Jahre (LASUÉN, 1973: 170). Gründe für 
diese beschleunigte Adoptionsgeschwindigkeit, welche sich in Zukunft eher noch verstärken dürfte, sind die verbesserten Transport- und Kommunikationsmittel und eine effizientere Integration und Anwendung von Ergebnissen aus der Forschung. Eine sich verstärkende Polarisierung ist das Ergebnis dieser zirkulär verursachten kumulativen Prozesse.

Um sein Modell auszubauen, hält Lasuén die Integration der Zentrale-Orte-Theorie in sein Wachstumspol-Konzept für unerläßlich. Sektorale Clusters beschränken sich demzufolge nicht mehr auf Industrien samt Input- und Output-Verbindungen, sondern berücksichtigen auch Dienstleistungsbereiche mitsamt ihren Verflechtungen. In sektoralen Clusters werden unterschiedliche Funktionen wahrgenommen. Gerade für Weltstädte dürften es vor allem Funktionen aus dem Dienstleistungsbereich sein, welche ihre führende Rolle in der Städtehierarchie determinieren.

\subsection{Die «World City Hypothesis» nach John Friedmann (1986)}

John Friedmann ist einer der wenigen Regionalwissenschaftler, der konzeptionell über Weltstadt-Systeme nachgedacht hat. Seine im Jahre 1986 veröffentlichte «World City Hypothesis» ist ein Versuch, den Zusammenhang zwischen Stadtentwicklung und weltwirtschaftlichen Einflußgrößen aufzuzeigen. Er hat dazu 7 Thesen formuliert.

Friedmann geht in seiner Abhandlung von der Hypothese aus, daß strukturelle Veränderungen innerhalb einer Stadt durch den Grad und die Form der Integration in die Weltwirtschaft sowie durch die der Stadt zugeordneten Funktionen innerhalb der neuen räumlichen Arbeitsteilung bestimmt werden. Er unterscheidet dabei exogene und endogene Faktoren, welche in verschiedener Art und Weise strukturelle Veränderungen in einer Stadt hervorrufen können. Zu den exogenen Faktoren zu zählen sind Richtung und Kapital der transnationalen Kapitalflüsse, die neue internationale Arbeitsteilung sowie die technologische Entwicklung. Räumliche Muster der geschichtlichen Akkumulation sowie die nationale Politik verstärken als endogene Faktoren die Wirkung der exogenen Faktoren.

Eine zentrale Stellung in der Argumentation von Friedmann nimmt das internationale Kapital ein. Friedmann stellt die These auf, daß Weltstädte die eigentlichen Zentren des internationalen Kapitals sind, da dieses in ihnen konzentriert und akkumuliert wird. Damit dienen die Weltstädte als Angelpunkte für eine komplexe räumliche Hierarchie.

Der Grad der Kontrollfähigkeit von Weltstädten über das interdependente Weltsystem wird in der Struktur und Dynamik ihrer Produktionssektoren reflektiert. Folgende Produktionssektoren resp. -segmente steuern dabei nach der Auffassung von Friedmann die Dynamik der Weltwirtschaft und somit auch der Weltstädte: Headquarter von multinationalen Unternehmen, internatio- nale Finanzunternehmen, weltweite Verkehrs- und Kommunikationsverbindungen, hochqualifizierte internationale Dienstleistungsbranchen (z. B. Werbebranche) sowie Einrichtungen mit nationalen oder internationalen Lenkungs- und Kontrollfunktionen (z. B. Uno-Headquarter) resp. mit kommunikativer Ausstrahlung (z. B. Messeplatz).

Friedmann ist sich allerdings bewußt, daß die Problematik der Weltstädte auch eine soziale Komponente hat. So stellt er beispielsweise fest, daß Weltstädte Ziel von binnen- und grenzüberschreitender Migration sind. Dies würde bedeuten, daß ein Zusammenhang zwischen (welt-)wirtschaftlichem Strukturwandel und den Migrationsbewegungen zu bestehen scheint. So vertritt Friedmann auch die Meinung, daß die in Weltstädten entstehenden sozialen Kosten höher sind als die fiskalische Kapazität dieser Städte.

\section{Ein neuer theoretischer Ansatz}

Ausgangspunkt der nachfolgenden Überlegungen bildet die These, daß für die Herausbildung von Weltstädten externe Effekte die entscheidende Rolle spielen. Weder räumliche Lagevorteile noch demographische Skalenerträge im Sinne von Urbanisationsvorteilen vermögen die Existenz einer Weltstadt hinreichend zu erklären.

Den Unterschied machen somit die Agglomerationsvorteile, d. h. der Saldo zwischen positiven und negativen externen Effekten, aus. Je größer die Differenz zwischen positiven und negativen Effekten, desto klarer und nachhaltiger sind die Chancen, eine Weltstadt ersten Ranges zu werden. Es verbleibt die Frage, wie sich kontinuierlich positive Saldi einzelner Städte erklären lassen. Die folgende Erklärung verknüpft vier Hauptfaktorfelder («Clusters») vor dem Hintergrund makroökonomischer und institutioneller Rahmenbedingungen:

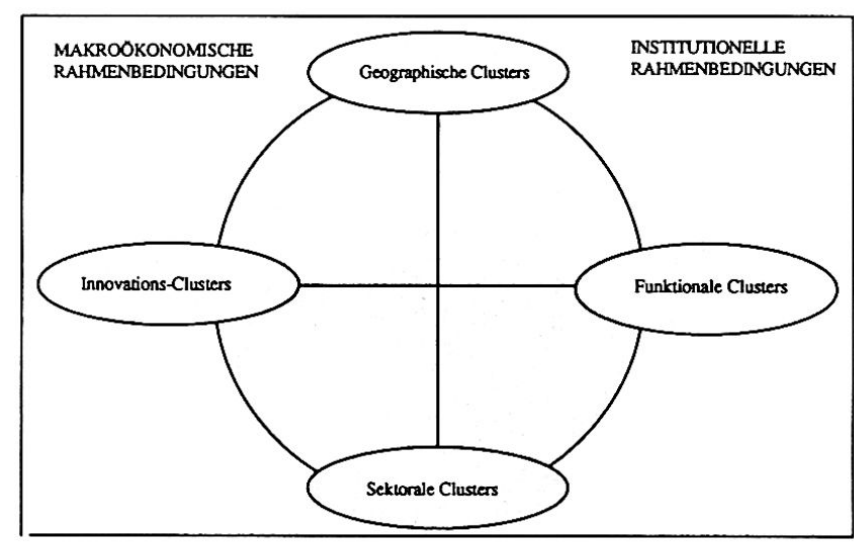

Abb. 3 Determinierende Einflußfaktoren der Stadtentwicklung. 
Stadtentwicklung ist ein dynamischer Prozeß. Sie spielt sich in einem Interaktionsfeld verschiedener "Clusters", die untereinander vernetzt sind, ab. Dieses Interaktionsfeld setzt sich aus Innovations-Clusters, geographischen, sektoralen und funktionalen Clusters zusammen. Eingebettet ist dieses Interaktionsfeld in makroökonomische und institutionelle Rahmenbedingungen. Stadtentwicklung verläuft in enger Abhängigkeit von diesen Rahmenbedingungen.

Verschiedene Begriffe dieses polarisationstheoretisch geprägten Stadtentwicklungsmodelles bedürfen einer Erklärung:

a) Cluster: Das Interaktionsfeld des Stadtentwicklungsprozesses wird von vier verschiedenen Clusters bestimmt. Der Begriff "Cluster» ist in diesem Zusammenhang, ähnlich wie bei Lasuén, als Anhäufung resp. gehäuftes Auftreten einer spezifischen Eigenschaft zu interpretieren.

Geographische Clusters sind in erster Linie als Ballung von Menschen zu verstehen. Ein gehäuftes Vorkommen von Menschen bedeutet in einem weiter gefaßten Sinn die Existenz eines Marktes. Märkte wiederum sind die eigentlichen Schauplätze jeglicher wirtschaftlicher Tätigkeit. Geographische Clusters können demnach als durch Infrastruktur gestaltete Räume mit Marktballung verstanden werden. Ein ausgeprägter geographischer Cluster kann auch extreme soziale Probleme mit sich bringen. Hohe Umweltschäden, das Drogenelend, eine bedrohliche Kriminalitätsrate oder eine zunehmende Verslumung können die Lebensqualität entscheidend beeinträchtigen. Die Schattenseiten der räumlichen Konzentration können die Vorteile, die durch die Ballung entstehen, mehr als wettmachen und die weitere Entwicklung einer Stadt hemmen, sofern die entsprechenden Kosten internalisiert werden.

Innovations-Clusters können als Standorte mit groBer Innovationsdynamik umschrieben werden. Ihr Auftreten hängt einerseits mit der Bevölkerungszahl zusammen, da das innovative Potential mit der Anzahl möglicher innovativ tätiger Akteure wächst. Andererseits benötigen Innovations-Clusters auch eine entsprechende, Innovationen fördernde Infrastruktur. Neben Universitäten und sonstigen höheren Forschungs- und Bildungsanstalten sind auch innovative Wirtschaftsbereiche - insbesondere hochwertige Dienstleistungen - zu dieser Infrastruktur zu zählen. Nicht zu vergessen ist in diesem Zusammenhang die formale und informale Kultur; sie schafft im weitesten Sinne die Voraussetzung für die Verbreitung einer «innovativen Atmosphäre».

Sektorale Clusters setzen sich aus Wirtschaftszweigen mit starken Kopplungseffekten zusammen. Vielfach vernetzte, sogenannte motorische Wirtschaftszweige vermitteln starke Wachstumsimpulse an die restlichen Wirtschaftszweige, indem sie entweder deren Produkte weiterverarbeiten (Rückwärtskopplung) oder Zwischenprodukte zur weiteren Verarbei- tung durch andere Unternehmungen herstellen (Vorwärtskopplung). Eine leistungsfähige Wirtschaft, in welcher ein Grundstock an qualitativ hochwertigen, exportfähigen Gütern produziert wird, schafft erst die Nachfrage nach hochspezialisierten Dienstleistungen. Der sektorale Cluster ist insofern eine Vorstufe des funktionalen Clusters.

Die funktionalen Clusters stellen eine Ergänzung des innovationstheoretischen Modelles sektoral und räumlich polarisierter Entwicklung nach Lasuén dar. Funktionale Clusters sind Ausdruck eines gehäuften räumlichen Auftretens spezifischer hochwertiger Funktionen für unternehmerische und institutionelle Entscheidungsprozesse. Im Falle einer Weltstadt sind dies die wichtigsten und global orientierten Lenkungsfunktionen, welche relativ stark vertreten sind.

b) "Handlungsdrehscheibe»: Die vier beschriebenen Clusters umreißen die "Handlungsdrehscheibe». Diese Drehscheibe verknüpft die sich zwischen den verschiedenen Clusters abspielenden Interaktionen. Für die Bedeutung einer Stadt ist somit nicht allein die große Bevölkerungszahl resp. der geographische Cluster entscheidend. Erst das Zusammenspiel von geographischen, sektoralen, funktionalen und Innovations-Clusters bringt die für die Stadtentwicklung entscheidende Dynamik hervor.

c) Makroökonomische Rahmenbedingungen: Stadtentwicklung hängt in starkem Maße vom makroökonomischen Umfeld ab. Komponenten dieses Umfeldes sind die üblichen Faktoren wirtschaftlicher Wettbewerbsfähigkeit und Stabilität, die Konjunktur, die Beschäftigungslage und die allgemeine Wirtschaftspolitik. Auch immaterielle Faktoren wie ein hohes Arbeitsethos oder eine überdurchschnittliche Leistungsbereitschaft der Bevölkerung beeinflussen die makroökonomischen Rahmenbedingungen in groBem Ausmaß. Das makroökonomische Umfeld muß insbesondere auf seine Dynamik hin untersucht werden. So kann in diesem Zusammenhang auch von «makroökonomischer Rahmen-Dynamik» gesprochen werden.

Bester Nährboden für eine gesunde urbane Entwicklung ist eine nachhaltig expandierende Wirschaft, deren Wachstum auf eigenen Anstrengungen und weltweit wettbewerbsfähigen Leistungen beruht. Diese Voraussetzungen sind insbesondere in den Städten der Dritten Welt nicht gegeben.

d) Institutionelle Rahmenbedingungen: Die Rolle der institutionellen, politischen und legalen Rahmenbedingungen ist mit der Rolle der makroökonomischen Rahmenbedingungen zu vergleichen. Die Wettbewerbsfähigkeit des institutionellen Umfeldes ist für die Stadtentwicklung ebenfalls von eminent großer Bedeutung.

Verschiedenste Komponenten gehören zu den institutionellen Rahmenbedingungen. Vertrauenswürdige Institutionen, eine gesicherte längerfristige Kontinuität, eine glaubwürdige Wirtschaftspolitik, juristische Sicherheit sowie respektierte Eigentumsrechte 
sind die wichtigsten unter ihnen. Von zunehmender Bedeutung ist auch eine hohe Entscheidungsfähigkeit. Die gegenwärtigen Produktionsprozesse und Entwicklungstrends verändern sich immer rascher und verlangen von den Behörden laufend schnellere Reaktionen auf veränderte äußere Einflüsse. Sind die notwendigen Entscheide einmal gefällt, muß zudem der Vollzug der getroffenen Maßnahmen sichergestellt sein. Entscheid und Vollzug werden in dem Ausmaß erschwert, als der wirtschaftliche und der politische Raum auseinanderfallen.

Erst wenn diese Rahmenbedingungen erfüllt sind, werden die für jegliches wirtschaftliche Wachstum unentbehrlichen Investitionen getätigt, stärkt sich der die wirtschaftliche Dynamik antreibende Unternehmergeist.

Die Verflechtungen im eben dargestellten Modell der Stadtentwicklung sind ausgeprägt. Für die «Handlungsdrehscheibe» wurden sie schon kurz angetönt. Auch auf der Ebene der Rahmenbedingungen besteht eine starke gegenseitige Abhängigkeit zwischen makroökonomischer und institutioneller Struktur und Dynamik.

Je besser die vier verschiedenen Clusters positiv zusammenspielen, je stärker sie dabei von institutionellen Rahmenbedingungen getragen und von makroökonomischen Rahmenbedingungen unterstützt werden, desto eher wird eine Stadt bzw. eine Agglomeration von weltweiter Bedeutung entstehen. Das Potential zur nach haltigen Kumulation der Wirkung der verschiedenen Clusters entscheidet über den Stellenwert einer Stadt. Nachhaltigkeit und Kontinuität jedoch hängen von der Qualität der Rahmenbedingungen ab.

\section{Empirische Befunde}

Die ersten empirischen Untersuchungen haben zahlreiche Indizien für die Relevanz des vorgestellten theoretischen Ansatzes geliefert. Um die theoretischen Aussagen stichhaltig nachzuweisen, wäre jedoch zweifelsohne zusätzliche empirische Arbeit zu leisten.

Es hat sich unter anderem bestätigt, daß das globale Städtesystem hierarchische Strukturen aufweist. An der Spitze der globalen Städtepyramide stehen die "Megastädte» Tokio, New York und London. In ihrer weltwirtschaftlichen Bedeutung überragen sie sämtliche anderen Städte und auch zahlreiche Nationalstaaten, indem sie in verschiedenen Bereichen Funktionen von globaler Tragweite übernehmen.

Eine zweite Stufe der Städtehierarchie wird von den «Makrostädten» eingenommen. Die von Makrostädten wahrgenommenen Funktionen entsprechen grundsätzlich denjenigen von Megastädten, umfassen jedoch weniger Bereiche und haben einen kleineren Wirkungsradius. Makrostädte sind schwieriger zu identifizieren, da der Wettbewerb zwischen bestehenden und potentiellen Makrostädten mit wachsender Internationalisierung $\mathrm{zu}-$ nimmt. Als heutige Makrostädte wurden Paris, Frank- furt, Los Angeles, San Francisco, Hongkong und Osaka identifiziert.

Auch in den Megastädten lassen sich zunehmende externe Kosten und eine rasch um sich greifende Wohlstandsmentalität, welche insgesamt entwicklungshemmend wirken, feststellen. Die geballte und verknüpfte Innovationskraft und Entscheidungsmacht in Tokio, New York und London erhalten jedoch deren Dynamik und festigen somit ihre Führungsposition in der globalen Städtehierarchie. Offensichtlich gelingt es diesen Städten, entstehende externe Kosten zumindest auf den weiteren Agglomerationsraum, teilweise aber auch auf die ganze $\mathrm{Na}$ tion zu überwälzen. Es scheint ein übergeordneter politischer Wille vorhanden zu sein, die Bedeutung dieser Metropolen weiter zu fördern.

Auch für die These, wonach ein ausgeprägter funktionaler Cluster für eine Weltstadt von zentraler Bedeutung ist, ließen sich empirische Indizien finden. Um den modernen Produktionsprozessen und Unternehmensformen und somit der heute vorherrschenden internationalen Arbeitsteilung gerecht zu werden, ist eine verstärkte Integration des funktionalen Aspektes in Stadtentwicklungsmodelle unumgänglich.

Institutionelle und makroökonomische Rahmenbedingungen sind, da sie durch die einzelnen Städte nur schwer zu verändern sind, Einflußgrößen einer höheren Stufe. Ihre Bedeutung dürfte, da sie schwer quantifizierbar sind, eher unter- denn überschätzt werden. Die vielfach stark subjektive Einschätzung vor allem der institutionellen Rahmenbedingungen erschwert die wissenschaftliche Erfaßbarkeit zusätzlich. Trotzem gibt es konkrete Hinweise, daß diese Rahmenbedingungen eine für jedes Stadtentwicklungs- und Raumordnungsmodell unumgängliche Einflußgröße bilden.

\section{Ausblick}

Die dargestellte theoretisch-konzeptionelle Durchdringung der Stadtentwicklungs-Problematik hat einerseits zu neuen Einsichten und zu einer verbesserten Ausgangslage für anschließende Analysen geführt. Andererseits hat sie, und dies ist unter Umständen höher einzuschätzen, die Sicht für weiterführende Fragestellungen geschärft:

- Eine erste Weiterführung der Analyse müßte die weltweite Bedeutung von Stadtsystemen vergleichen und damit die üblicherweise punktuelle Sichtweise ergänzen. Wahrscheinlich ist die Konkurrenzfähigkeit einer Stadt nicht nur von ihrer eigenen Dynamik und jener ihrer Agglomeration abhängig, sondern zusätzlich von der Attraktivität des regionalen (allenfalls nationalen und internationalen) Stadtsystems, dem sie angehört. Je kleinräumiger und je dichter besiedelt ein Land, desto eher dürften diese Überlegungen zutreffen. Die Erfahrungen bei der Verfassung der Nationalfonds-Studie haben gezeigt, daß die methodischen und empirischen Probleme bei einer solchen Maßstabserweiterung exponentiell zunehmen dürften. 
Dennoch könnte sich ein erster Schritt in dieses wissenschaftliche Neuland lohnen.

- Der zweite vorzusehende Fragebereich umfaßt die vielfältigen Aspekte von Externalitäten großer Städte. Eine vorwiegend wirtschaftliche Sichtweise klammert ökologische und soziale Aspekte der Stadtentwicklung weitgehend aus. Diese wären jedoch einzufügen, da dynamisches urbanes Wachstum in aller Regel eben nicht nur positive Externalitäten (z. B. im Bereich der Infrastruktur und Volkswirtschaft), sondern auch negative Externalitäten (vor allem im Umwelt- und Sozialbereich) verursacht. Die naheliegende erste Folgerung ist, daß gerade Mega- und Makrostädte erheblich mehr negative als positive Externalitäten verursachen, die letztlich entweder durch die außerhalb der Stadt lebenden Steuerzahler bezahlt werden müssen oder die einen zunehmenden Verlust an Lebensqualität in den betroffenen Städten bewirken. Beide Mechanismen führen über kurz oder lang zu nachteiligen Effekten für die betreffende Stadt. Nun gibt es aber - und dies ist eine zweite Folgerung - Weltstädte, welche offenbar im größeren nationalen Kontext Einigkeit darüber erreichen, daß die anfallenden negativen Externalitäten bei weitem durch die positiven Externalitäten im wirtschaftlichen und infrastrukturellen Bereich aufgewogen, sozusagen überkompensiert werden. Der Saldo wird für das Gesamtsystem als deutlich positiv perzeptiert - und deshalb werden solche makroökonomische und institutionelle Rahmenbedingungen geschaffen bzw. weiter aktiv gepflegt, die der entsprechenden Stadt weiterhin die für eine nachhaltige Entwicklung notwendige innere und äußere Dynamik erlauben.

So wären bei weiterführenden Arbeiten letztlich zwei Aspekte auseinanderzuhalten: einerseits die dynamische Saldo-Betrachtung bezüglich positiver und negativer Externalitäten für Weltstadt-Systeme auf der Mega-, Makro- und Meso-Ebene; andererseits die Beurteilung und Internalisierung dieses Saldos durch Rahmenbedingungen, die durch lokale und nationale Politik weitestge- hend bestimmt werden. Die politökonomische Analyse der Ausgestaltung von stadtrelevanten Rahmenbedingungen müßte dabei idealerweise die ökonomische Analyse der Externalitäten ergänzen.

\section{Literatur:}

BRUGGER, E. A. (1987): "Multinational Enterprises between Functionality and Territoriality", presented at: European Science Foundation - Workshop on World Economy and spatial Organisation of Power: The Dynamics of Core-Periphery Relations, Jerusalem, in Druck.

BRUGGER, E. A., KÄRCHER, T. (1992): Weltstädte - Schweizer Städte: Die Funktion schweizerischer Großstädte im weltwirtschaftlichen Kontext. In: Bericht 5 des NFP "Stadt und Verkehrn, Zürich.

FREY, R. (1990): Städtewachstum - Städtewandel, Basel.

FRIEDMANN, J. (1986): "The World City Hypothesis». In: Development and Change (SAGE, London, Beverly Hills and New Delhi), Vol. 17, S. 69-83.

LASUÉN, J. (1973): “Urbanization and Development - The Temporal Interaction between Geographical and Sectoral Clusters". In: Urban Studies, Vol. 10.

Richardson, H. W. (1978): Urban Economics, Hinsdale-Illinois.

SCHÄTZL, U. (1981): Wirtschaftsgeographie 1, Paderborn.

SCHILLING-KALETSCH， I. (1976): "Wachstumspole und Wachstumszentren - Untersuchungen zu einer Theorie sektoral und regional polarisierter Entwicklung". In: Arbeitsberichte und Ergebnisse zur wirtschafts- und sozialgeographischen Regionalforschung 1, Wirtschaftsgeographische Abteilung des Instituts für Geographie und Wirtschaftsgeographie der Uni Hamburg.

STIGLBAUER, K. (1989): “Neue Rollen der Großstädte in hochentwickelten Staaten - Wien als Beispiel». In: Stadtentwicklung im Wandel technologischer und sozio-ökonomischer Rahmenbedingungen, Rhein-Mainische Forschungen, Heft 103, Frankfurt a. M.

WACHTER, D. (1990): Externe Effekte, Umweltschutz und regionale Disparitäten, Zürich. 